\title{
Mitgliederversammlung (1. Teil)
}

Präsident Prof. Dr. K. Kremer, Düsseldorf: Meine sehr verehrten Damen und Herren, ich eröffne den ersten Teil der Mitgliederversammlung und darf feststellen, daß alle Mitglieder unserer Gesellschaft termingerecht eingeladen worden sind. Die Einladung ist Ihnen mit Heft 2/1976 der Mitteilungen unserer Gesellschaft zugegangen.

\section{Totenehrung}

Es gehört zu den traurigen Pflichten des Präsidenten, der Chirurgen zu gedenken, die unserer Gesellschaft angehörten und seit unserer letzten Tagung von uns gingen. Wir haben den Tod von $43 \mathrm{Mitglie}-$ dern zu bedauern, denen wir unserer letzte Reverenz erweisen wollen. Ich nenne die Namen in der Folge ihres Ablebens:

Kurt Götte, Bad Hersfeld Paul Gohrbandt, Berlin Peter Riess, Solingen Hans Radig, Dortmund-Hombruch Horst Richard Lewinski, Ehringshausen

Karl Ewald Herlyn, Göttingen-Weende Franz Merke, Basel

Rudolf Bertram, Gelsenkirchen-Horst Heinz Junge, Sande/Oldenburg

Enno Willms, Sulingen Herbert Kraus, Wien Hartwig Eggers, Magdeburg

Hans May, Philadelphia - Korrespondierendes Mitglied - Herbert Schoen, Karlsruhe

Lester Dragstedt, Florida - Korrespondierendes Mitglied -

Andreas Kosinzew, Garmisch-Partenkirchen Franz Gillitzer, Düsseldorf

Friedrich Haas, Innsbruck Hans Zimmer, Dresden Mas Saegesser, Thun

Max Lange, München Kurt Herzog, Düsseldorf

Michael Paraskevas, Athen - Korrespondierendes Mitglied - $\quad$ Erich Marsch, Berlin-Moabit

Dietrich Cassau, Berlin Werner Jantke, Duisburg-Buchholz

Paul Zsigmond, Frankfurt-Niederrad Walter Neussel, Hagen Paul Huber, Innsbruck

German Eichelter, Wien Arthur Weiss, Berlin Otto Henningsen, Rom

Walther Künster, Koblenz Ernst Wegener, Bremen-Vegesack

Robert Kampshoff, Essen-Borbeck Wilhelm Wagner, Bad Harzburg

Hans Küster, Hamburg-Altona Elisabeth Jungermann, Zwischenahn

Eugen Müller, Nürtingen Curt Göcke, Dresden Klaus Kuhlmann, Aachen

Besonders schmerzlich empfinde ich es, Sie vom Tod unseres erst im Vorjahr ernannten Ehrenmitgliedes Hans Hellner unterrichten zu müssen. Wenn er auch in den letzten Jahren aus gesundheitlichen Gründen unseren Tagungen fernblieb, so ist er uns doch dank der eindrucksvollen Laudatio seines Schülers Gelbke, unseres Präsidenten des Jahres 1973, in guter Erinnerung. Hohe Intelligenz, fachlicher Weitblick, allgemeine Fairne $B$ und menschliche Toleranz zeichneten ihn aus. Wir werden seiner stets in Hochachtung gedenken.

Am 9. März 1976 verstarb auch unser langjähriger Wahlleiter Herr Professor Ewald Weisschedel nach schwerer Krankheit. Ihnen.

Ich darf Sie bitten, sich zum Gedenken unserer Toten von den Plätzen zu erheben. - Ich danke 